Résumés des conférences et travaux

\title{
Histoire des sciences au Moyen Âge
}

Danielle Jacquart

\section{OpenEdition \\ Journals}

\section{Édition électronique}

URL : https://journals.openedition.org/ashp/707

DOI : 10.4000/ashp.707

ISSN : 1969-6310

\section{Éditeur}

Publications de l'École Pratique des Hautes Études

\section{Édition imprimée}

Date de publication : 1 octobre 2009

Pagination : 177-179

ISSN : 0766-0677

\section{Référence électronique}

Danielle Jacquart, "Histoire des sciences au Moyen Âge », Annuaire de l'École pratique des hautes études (EPHE), Section des sciences historiques et philologiques [En ligne], 140 | 2009, mis en ligne le 29 octobre 2009, consulté le 06 juillet 2021. URL : http://journals.openedition.org/ashp/707 ; DOI : https://doi.org/10.4000/ashp.707 


\title{
HISTOIRE DES SCIENCES AU MOYEN ÂGE
}

\author{
Directeur d'études : $\mathrm{M}^{\mathrm{me}}$ Danielle JACQUART
}

Programme de l'année 2007-2008 : I. L'intérêt pour les sciences durant le haut Moyen Âge. II. Alchimie et médecine aux XIV et XV $V^{e}$. - III. Les collections manuscrites de textes scientifiques : constitution et évolution.

La rentrée a été endeuillée par le décès de Guy Beaujouan, premier titulaire de la direction d'études « Histoire des sciences au Moyen Âge », survenu le 5 octobre 2007. Une séance d'hommage, à laquelle ont participé activement Nicolas Weill-Parot et Matthieu Husson, a été consacrée à son œuvre.

\section{L'intérêt pour les sciences durant le haut Moyen Âge}

Poursuivant les travaux entrepris l'année précédente - qui avaient essentiellement porté sur l'œuvre de Bède - nous avons ouvert le chapitre des réalisations de l'époque carolingienne. En une première étape nous avons réévalué la place qu'occupent dans les curiosités d'un Alcuin les sciences de la nature et les mathématiques. Si ses Versus de patribus, regibus et sanctis Euboricensis ecclesiae font une place non négligeable à l'étude des sciences de la nature dans leur description des intérêts intellectuels de l'école cathédrale d'York, où il reçut sa formation, Alcuin se montre lui-même plus attiré par la science du nombre. En astronomie, où il est très dépendant de Bède, c'est le versant arithmétique qui le retient surtout, avec le souci de retrouver les nombres que Dieu a mis dans le monde qu'il a créé. Dans ce contexte mathématique, nous ne pouvions éviter d'analyser quelques problèmes des Propositiones ad acuendos iuvenes, dont l'attribution à Alcuin est désormais retenue par les spécialistes.

Après cette brève évocation de la figure d'Alcuin, c'est au Periphyseon de Jean Scot que nous nous sommes consacrés, en recherchant la place qu'y tiennent les arts du quadrivium et le rôle que ceux-ci jouent dans le système philosophique de l'Érigène. Les arts libéraux sont énumérés et définis quand il est question au livre I de la catégorie du lieu, à propos de laquelle le disciple conclut « qu'il n'y a de lieu que dans l'esprit ». L'arithmétique définie en ce livre I comme « la discipline constante et sans tache des nombres soumis à l'examen attentif de l'esprit » est dite au livre III « fondement immobile, cause primordiale et principe des autres arts ». Les exposés d'arithmétique, dont beaucoup sont inspirés de Boèce, ne sont là que pour expliquer comment passer des nombres éternels procédant de la monade aux nombres dont a connaissance l'esprit humain et qui fondent donc l'arithmétique. Ces exposés sont indissociables d'une explicitation des processus cognitifs, la mémoire formant le lieu de confluence des nombres intelligibles, émanations dans l'intellect des nombres éternels, et des species des choses sensibles saisies par les sens corporels, ainsi que le résume le disciple : 
« Tu as entrepris, à ce que je crois, de persuader que tous les nombres émanant de la monade comme d'une source s'écoulent, comparables à deux rivières issues d'un même courant d'eau mais dans deux lits séparés, dont l'une court à travers les canaux internes de la nature (c'est-à-dire à travers l'intellect et la raison), l'autre à travers les apparences extérieures des choses visibles et les sens, jusqu'à ce qu'elles confluent dans la mémoire » (Periphyseon, livre III, 660D-661C).

Dans le cadre néo-platonicien qui est celui du Periphyseon, les notions de participation et d'ordre naturel déterminent la représentation cosmologique. C'est dans cette perspective qu'il faut situer les allusions héliocentristes de Jean Scot, mises en avant par les historiens de l'astronomie depuis Pierre Duhem, ces allusions n'excluant d'ailleurs pas l'attribution d'une position centrale et immobile à la terre. Tant dans les gloses aux Noces de Philologie et Mercure que dans le Periphyseon il est en effet énoncé que Jupiter, Mars, Vénus et Mercure tournent autour du soleil. Dans le Periphyseon cette proposition est faite au sein d'un long développement tendant à montrer qu'il n'y a pas d'eaux au-dessus du firmament et que la pâleur des étoiles les plus éloignées de la terre n'est pas due à la présence de l'élément aqueux, mais à l'absence de chaleur. Saturne qui est proche « de la ronde des étoiles » est dite froide et pâle. Selon le même raisonnement les planètes Jupiter, Mars, Vénus et Mercure, qui parcourent leurs cercles autour du soleil, montrent des faces claires quand elles sont audessus de celui-ci et des faces rouges quand elles sont au-dessous. C'est plus un ordre du monde tant spirituel que physique qu'une représentation cosmologique qu'entreprend de livrer Jean Scot. Les longues digressions sur les distances entre la terre et la lune ou entre la terre et le soleil, si elles font appel à des données techniques fondées sur une ample information livresque, n'en visent pas moins à retrouver à travers des calculs complexes la raison des nombres. Quant aux rares développements sur la géométrie, ils révèlent surtout une réflexion approfondie sur ce que sont les objets géométriques. En résumé, dans notre investigation sur « les intérêts scientifiques » manifestés par les auteurs du haut Moyen Âge, Jean Scot Érigène se signale par l'intégration de ceux-ci dans un projet philosophique, plus attentif aux processus cognitifs qu'à l'explication des phénomènes.

\section{Alchimie et médecine aux $X I V^{e}$ et $X V^{e} s$.}

En prélude à la reprise des travaux sur les rapports entre alchimie et médecine, le livre de Didier Kahn, Alchimie et paracelsisme en France à la fin de la Renaissance (1567-1625), paru en 2007 chez Droz dans la collection des «Cahiers d'Humanisme et Renaissance », nous a servi de guide pour revenir sur des questions fondamentales telles que la définition (ou plutôt les définitions) de l'alchimie, ses différents courants, son évolution jusqu'à l'œuvre de Paracelse, déterminante pour ses rapports avec la médecine. L'étude a porté ensuite sur la notion de quintessence, son origine et son développement, ainsi que sur l'eau ardente, en commençant par la lecture de passages du De consideratione quintae essentiae rerum omnium de Jean de Roquetaillade, écrit vraisemblablement vers 1351-1352. Nous nous sommes particulièrement attachés au chapitre de la distillation de l'antimoine, ainsi introduit par son auteur : 
« Dieu lui-même est le témoin céleste et béni que maintenant je vais te révéler un très grand secret qui n'a jamais été livré par personne, ou est connu de très peu, et c'est le secret de tous les secrets : extraire la quintessence de l'antimoine ».

Si une addition dans l'édition de Gratorolo (Bâle 1561, rééd. 1597) précise, à la fin du chapitre, que cette préparation « traite en un temps court tous les apostumes, si elle est apposée dessus à la mode d'un onguent », le texte de Rupescissa lui-même ne donne aucune indication sur son mode d'utilisation médicale. Cette imprécision mérite d'être notée lorsqu' on la replace dans le cadre de « la querelle de l'antimoine » qui a agité les milieux médicaux, notamment parisiens, à partir de la fin du $\mathrm{XvI}^{\mathrm{e}}$ siècle : elle portait justement sur l'usage interne de cette substance et ses effets toxiques, l'usage externe n'était pas en cause. Si nous nous sommes fondés sur l'édition de Gratarolo, nous avons aussi recouru au manuscrit latin 7151 de la Bibliothèque nationale de France. À cette occasion, le directeur d'études est revenu sur la datation généralement proposée : ce manuscrit n'est pas du XIV $v^{e}$ siècle, mais du XV siècle et d'origine milanaise. Nous avons terminé l'année en analysant le De aqua ardente de Michel Savonarole, écrit en latin en 1440 à la demande du juriste Antonio Roselli, et traduit en italien à l'intention de Leonello d'Este entre 1444 et 1450 . Fidèle à sa confiance dans les bienfaits du vin, dont les effets sont mis en rapport avec le rôle du soleil dans la maturation des raisins, Michel Savonarole ne prend en considération que l'eau-devie distillée à partir de celui-ci. En grande partie compilation d'ouvrages antérieurs, dont les Consilia de Taddeo Alderotti, le traité de Michel Savonarole manifeste aussi des préoccupations médicales et sociales. La vogue de l'eau-de-vie dans les villes de l'Italie du Nord de cette première moitié du $X^{e}{ }^{e}$ siècle fait apparaître un danger qui ne porte pas encore son nom, l'accoutumance alcoolique. La perception de ce danger, jointe à la difficile intégration des effets de l'eau ardente dans la doctrine galénique, explique peut-être que Michel Savonarole ne mentionne pas ce produit dans ses ouvrages proprement médicaux.

Dans le cadre de ce thème «Alchimie et médecine », nous avons poursuivi l'analyse de l'opuscule sur la lithiase d'Angelo d'Aquila (voir Livret-Annuaire, 2005-2006, p. 214 et Annuaire, 2006-2007, p. 155). Nous avons à nouveau relevé la manière étrange dont Angelo utilise ses sources, mais nous n'avons pu parvenir encore à percer le mystère de ce traité déroutant.

\section{Les collections manuscrites de textes scientifiques : constitution et évolution}

Dans la perspective du colloque international sur l'école de Saint-Victor (2425 septembre 2008), les travaux sur les collections manuscrites de textes scientifiques ont porté sur le fonds victorin à la Bibliothèque nationale de France. Il était nécessaire d'exposer en préambule aux auditeurs l'histoire de la bibliothèque de Saint-Victor et des méthodes de classement dont portent la trace les manuscrits. L'édition du catalogue de Claude de Grandrue (1514) par Gilbert Ouy (Brepols, 1999) nous a évidemment servi de base. Avec l'ancien fonds de Saint-Victor, c'est l'exemple de recueils factices reflétant essentiellement des exigences de bibliothécaires qui était illustré de 
manière extrême. Les résultats obtenus seront publiés par le directeur d'études dans les actes du colloque. Ils portent principalement sur les manuscrits à contenu astronomique et astrologique dont des témoins majeurs étaient présents dans le fonds dès le $\mathrm{XII}^{\mathrm{e}}$ siècle. Nous avons remarqué la proportion importante des pertes d'exemplaires à contenu astrologique depuis la rédaction du catalogue de Claude de Grandrue. Des pertes significatives touchent de manière concomitante des textes d'alchimie et de magie. Plusieurs indices inclinent à y voir plutôt la manifestation de convoitises de la part de lecteurs indélicats que l'effet d'une quelconque censure, d'autant plus que des acquisitions de nouveaux manuscrits astrologico-magiques étaient effectuées dans les mêmes années. 